\title{
Special Issue on Bioinformatics and Machine Learning for Cancer Biology
}

\author{
Shibiao Wan ${ }^{1, *} \mathbb{C}$, Chunjie Jiang ${ }^{2} \mathbb{O}$, Shengli $\mathrm{Li}^{3}$ and Yiping Fan ${ }^{1}$ \\ 1 Center for Applied Bioinformatics, St. Jude Children's Research Hospital, Memphis, TN 38105, USA; \\ yiping.fan@stjude.org \\ 2 Division of Diabetes, Endocrinology and Metabolism, Department of Medicine, Baylor College of Medicine, \\ Houston, TX 77030, USA; chunjie.jiang917@outlook.com \\ 3 School of Medicine, Shanghai Jiao Tong University, Shanghai 201620, China; shengli.li@shsmu.edu.cn \\ * Correspondence: shibiao.wan@stjude.org; Tel.: +1-901-595-1905
}

check for updates

Citation: Wan, S.; Jiang, C.; Li, S.; Fan, Y. Special Issue on Bioinformatics and Machine Learning for Cancer Biology. Biology 2022, 11, 361. https:// doi.org/10.3390/biology11030361

Received: 15 February 2022 Accepted: 23 February 2022 Published: 24 February 2022

Publisher's Note: MDPI stays neutral with regard to jurisdictional claims in published maps and institutional affiliations.

Copyright: (C) 2022 by the authors. Licensee MDPI, Basel, Switzerland. This article is an open access article distributed under the terms and conditions of the Creative Commons Attribution (CC BY) license (https:// creativecommons.org/licenses/by/ $4.0 /)$.
Cancer is a leading cause of death worldwide, claiming millions of lives each year. Cancer biology is an essential research field to understand how cancer develops, evolves, and responds to therapy. By taking advantage of a series of "omics" technologies (e.g., genomics, transcriptomics, and epigenomics), computational methods in bioinformatics and machine learning can help scientists and researchers decipher the complexity of cancer heterogeneity, tumorigenesis, and anticancer drug discovery. Particularly, bioinformatics enables the systematic interrogation and analysis of cancer from various perspectives, including genetics, epigenetics, signaling networks, cellular behavior, clinical manifestation, and epidemiology. Moreover, thanks to the influx of next-generation sequencing (NGS) data in the postgenomic era and multiple landmark cancer-focused projects, such as The Cancer Genome Atlas (TCGA) and Clinical Proteomic Tumor Analysis Consortium (CPTAC), machine learning has a uniquely advantageous role in boosting data-driven cancer research and unraveling novel methods for the prognosis, prediction, and treatment of cancer.

This special issue aims to leverage bioinformatics analysis and machine learning to further our understanding of cancer biology in different perspectives. Specifically, Yao et al. [1] identified and validated an Annexin-related prognostic signature and therapeutic targets for bladder cancer. Furthermore, for bladder cancer, Wei et al. [2] demonstrated CPA4 to be a poor prognostic biomarker correlated with immune cells infiltration, and for ovarian cancer, Li et al. [3] identified the RNA modification gene PUS7 as a potential biomarker. Another interesting development is that Serna-Blasco et al. [4] proposed a new measurement called R-score to assess the quality of variants' calls using liquid biopsies for non-small cell lung cancer. Additionally, for breast cancer, Zainab et al. [5] used a drug-drug interaction network approach to identify estrogen receptor alpha inhibitors, and simultaneously, they revealed the role of persistent organic pollutants in the progression of the breast cancer. Furthermore, Chiu et al. [6] identified a DNA damage repair gene set as a potential biomarker to stratifying patients with high tumor mutational burden. Methodically, Rehman et al. [7] proposed a depth-wise convolutional neural network for architecture distortion-based digital mammograms classification. Obermayer et al. [8] proposed a web-based framework called DRPPM-EASY for integrative analysis of multiomics cancer datasets.

It is exciting to know that, with the help of integrative bioinformatics analyses, our understanding of multiple cancers such as bladder cancer, ovarian cancer, breast cancer, and non-small cell lung cancer has been remarkably enhanced. Additionally, with the application of machine learning approaches (especially deep learning) and webtool development, our capabilities of extending the analysis and understanding of other less-studied cancers are expected to be consolidated. On the other hand, we are also aware that with heterogeneity and complexity properties, even the internal mechanisms of tumorigenesis 
for those well-studied cancers $[9,10]$ remain to be further unraveled, let alone the discovery of anticancer drugs and treatment. More integrative, genome-wide, and global-scale studies are required to further shed light on the driving forces behind the tumorigenesis and the development of anticancer drugs and treatments.

In summary, we would like to thank all the authors for the articles published within this special issue who made significant contributions to this special issue, and more importantly, to the better understanding of various cancers as well as cancer-related analysis method improvements. We are eager to see more exciting discoveries in cancer biology with the help of bioinformatics analysis and machine learning in the near future.

Author Contributions: S.W. wrote the main manuscript. S.W., C.J., S.L. and Y.F. participated in revising the manuscript. All authors have read and agreed to the published version of the manuscript.

Funding: This research was funded by in part by the National Cancer Institute grant P30 CA021765. The content is solely the responsibility of the authors and does not necessarily represent the official views of the National Institutes of Health. This work was in part supported by National Natural Science Foundation of China (32100517) and Shanghai General Hospital Startup Funding (02.06.01.20.06).

Conflicts of Interest: The authors declare no conflict of interest. The funders had no role in the design of the study; in the collection, analyses, or interpretation of data; in the writing of the manuscript, or in the decision to publish the results.

\section{References}

1. Yao, X.; Qi, X.; Wang, Y.; Zhang, B.; He, T.; Yan, T.; Zhang, L.; Wang, Y.; Zheng, H.; Zhang, G.; et al. Identification and Validation of an Annexin-Related Prognostic Signature and Therapeutic Targets for Bladder Cancer: Integrative Analysis. Biology 2022, 11, 259. [CrossRef]

2. Wei, C.; Zhou, Y.; Xiong, Q.; Xiong, M.; Hou, Y.; Yang, X.; Chen, Z. Comprehensive Analysis of CPA4 as a Poor Prognostic Biomarker Correlated with Immune Cells Infiltration in Bladder Cancer. Biology 2021, 10, 1143. [CrossRef] [PubMed]

3. Li, H.; Chen, L.; Han, Y.; Zhang, F.; Wang, Y.; Han, Y.; Wang, Y.; Wang, Q.; Guo, X. The Identification of RNA Modification Gene PUS7 as a Potential Biomarker of Ovarian Cancer. Biology 2021, 10, 1130. [CrossRef] [PubMed]

4. Serna-Blasco, R.; Sánchez-Herrero, E.; Berrocal Renedo, M.; Calabuig-Fariñas, S.; Molina-Vila, M.Á.; Provencio, M.; Romero, A. R-Score: A New Parameter to Assess the Quality of Variants' Calls Assessed by NGS Using Liquid Biopsies. Biology 2021, 10, 954. [CrossRef] [PubMed]

5. Zainab, B.; Ayaz, Z.; Rashid, U.; Al Farraj, D.A.; Alkufeidy, R.M.; AlQahtany, F.S.; Aljowaie, R.M.; Abbasi, A.M. Role of Persistent Organic Pollutants in Breast Cancer Progression and Identification of Estrogen Receptor Alpha Inhibitors Using In-Silico Mining and Drug-Drug Interaction Network Approaches. Biology 2021, 10, 681. [CrossRef] [PubMed]

6. Chiu, T.-Y.; Lin, R.W.; Huang, C.-J.; Yeh, D.-W.; Wang, Y.-C. DNA Damage Repair Gene Set as a Potential Biomarker for Stratifying Patients with High Tumor Mutational Burden. Biology 2021, 10, 528. [CrossRef] [PubMed]

7. Rehman, K.U.; Li, J.; Pei, Y.; Yasin, A.; Ali, S.; Saeed, Y. Architectural Distortion-Based Digital Mammograms Classification Using Depth Wise Convolutional Neural Network. Biology 2022, 11, 15. [CrossRef] [PubMed]

8. $\quad$ Obermayer, A.; Dong, L.; Hu, Q.; Golden, M.; Noble, J.D.; Rodriguez, P.; Robinson, T.J.; Teng, M.; Tan, A.-C.; Shaw, T.I. DRPPMEASY: A Web-Based Framework for Integrative Analysis of Multi-Omics Cancer Datasets. Biology 2022, 11, 260. [CrossRef]

9. Singh, S.; Quarni, W.; Goralski, M.; Wan, S.; Jin, H.; Van de Velde, L.A.; Fang, J.; Sing, R.; Fan, Y.; Johnson, M.; et al. Targeting the Spliceosome through RBM39 Degradation Results in Exceptional Responses in High-Risk Neuroblastoma Models. Sci. Adv. 2021, 7, 47, eabj5405. [CrossRef]

10. Wang, R.; Zheng, X.; Wang, J.; Wan, S.; Song, F.; Wong, M.H.; Leung, K.S.; Cheng, L. Improving Bulk RNA-seq Classification by Transferring Gene Signature from Single Cells in Acute Myeloid Leukemia. Brief. Bioinform. 2022, bbac002. [CrossRef] [PubMed] 